\title{
CONTRACTS, COST SHARING AND CONSISTENCY
}

\author{
MAURICE KOSTER
}

\begin{abstract}
Under a contract, agents are not only held to honor the allocation as prescribed by a cost sharing mechanism but also a full description of allocated units and costs once production falls short. For agents leaving the cost sharing problem by taking their demanded units and prepaying the corresponding bill, a contract allows for a reformulation of the cost sharing problem to serve the remaining agents. Consistency requires invariance of cost shares relative to any such reduced cost sharing problem. Under consistency, the proportional mechanisms uniquely satisfy additivity and positivity of cost shares. Exchanging positivity by equal treatment characterizes the set of mechanisms which propose proportional shares for only those agents in the maximal indifference set for some preordering on the rest of nonnegative numbers. This includes egalitarian and average cost sharing. The latter is further characterized by the properties linearity. Under $R$-consistency, a mechanism is supported by at least one reasonable contract which meets upperbounds. The class of additive and $R$-consistent mechanisms is isomorphic to the class of consistent and monotonic rationing methods. Consequently serial cost sharing is R-consistent, whereas Shapley-Shubik is not. Examples are given how the extensive literature on consistent monotonic rationing can be inferred to study and characterize cost sharing mechanisms.
\end{abstract}

Keywords: Cost sharing; Consistency; Additivity; Constant returns; Rationing; (Quasi-) Proportional mechanism; Egalitarian mechanism; Serial mechanism; Shapley-Shubik mechanism

\section{JEL-Classification: C70, D63, D70}

\section{INTRODUCTION}

This paper fits in the stream of axiomatic cost sharing literature (see Thomson (2001), Moulin (2002)) which discusses structural and characterizing properties of solutions. I will consider homogeneous cost sharing problems; a group of agents $N$ have demands $q=\left(q_{i}\right)_{i \in N}$ for a single perfectly divisible output and corresponding production $\operatorname{costs} C\left(\sum_{i \in N} q_{i}\right)$ have to be shared. Central property is that of consistency, which is studied in various fields in economics and social choice theory as a fundamental principle to decision problems pertaining to varying

Acknowledgements: I thank Hervé Moulin, Justin Leroux, Juan Moreno-Ternero and two anonymous referees for many useful comments. The main ideas for this paper were developed when I was at NPS, Monterey, and Rice University. Financial support by these institutes is gratefully acknowledged. 
sets of agents. In the cost sharing context, the basic idea is the following: Suppose that agent $i$ leaves the cost sharing problem, by taking away the demanded units $q_{i}$ and prepaying his cost share $\mu_{i}(q, C)$. In line with much of the literature then a solution $\mu$ is called consistent if the calculated cost shares for the remaining agents in $N \backslash i$ in the reduced cost sharing problem $\left(q_{N \backslash i}, C^{*}\right)$ are the same, or $\mu_{N \backslash i}(q, C)=\mu\left(q_{N \backslash i}, C^{*}\right)$; the reduced cost function $C^{*}$ ideally presents the cost structure after $i$ has gone, therebye using the information contained in the model given by $(q, C)$ and $\mu$. Following Young (1985, p19), this self-similarity property envisions an idea of fairness of solutions at all levels of cooperation, for any subgroup of agents, according to which 'no subgroup should want to "re-contract"'.

Where for some models in the distributive justice literature, like the rationing model, the concept of consistency is transparent and unambiguous, for the (homogeneous) cost sharing problem it is not. The problems do not arise in choosing the reduced demand profile, which is obviously $q_{N \backslash i}$, but in choosing an appropriate reduced cost function $C^{*}$. In the literature several proposals have been made, each serving its own purpose. Below I will argue that there is still a clear issue with defining consistency in homogeneous cost sharing problems, and contribute to the literature with a new proposal.

First I will extend the notion of a cost sharing solution by that of a contract, which also specifies the way produced units and corresponding costs are shared in case demand is not fully met. Each contract is used to define a reduced cost function, and a reduced cost sharing problem in particular. I will call a solution consistent if for all possible contracts the agents in the reduced cost sharing problem need not renegotiate since the solution calculates the same cost shares as before. A solution is weakly consistent if there exists at least one contract supporting it accordingly. So the basic feature of a consistent solution - under the weak or strong notion

- is that it offers the agents an opportunity to agree to a contract which rationalizes the cost shares at hand, by implementing the same solution for all contingent reductions based on this contract. If no such contract exists, there is the intrinsic problem of agents holding different opinions to which reduced problem the solution must be applied.

1.1. Relation to the literature. Basically the efforts to properly define consistency in cost sharing problems can be divided in two branches, each of which originated from notions of consistency for cooperative games due to Davis and Maschler (1965), Sobolev (1973), and Hart and Mas-Collel (1989). The fundamental difference is the way that reduced costs are modeled. First, the residual cost put forward by the literature à la Hart and Mas-Colell is the total cost minus the total amount the complement subgroup of remaining agents would have to pay under the cost sharing solution, where potential revision of the demands of the remaining agents is incorporated. Leroux (2007) suggests a definition in this fashion for the model discussed here, but needs solutions fit for heterogeneous cost functions. Sudhölter (1998) 
applies the original ideas to cooperative games associated with cost sharing problems. However, in light of contingent externalities it is not clear which transformation of a cost sharing problem into a cost game fits best.

The second branch of literature, based on ideas of Davis and Maschler (1965), treats the residual costs as the total cost minus what the complement coalition has already paid for. Examples are Moulin and Shenker (1994), Kolpin (1994), Tijs and Koster (1998), and Albizuri and Zarzuelo (2007). Again, these papers show the richness of the model allows for many types of reduction, each expressing a different notion of consistency.

This paper contributes to the literature in the following ways. First, the arbitrariness in choosing the appropriate reduction is to some degree tackled by requiring invariance of cost shares to sets of reductions. Second, although one could argue the paper belongs to the Hart and Mas-Collel type of literature, the notion of a contract takes into account a continuum of hypothetical cost sharing problems.

1.2. Overview of the paper and results. Section 2 provides the basic model of homogeneous cost sharing, as well as the notion of solution in these contexts. Section 3 introduces the basic notions of contract, reduced cost sharing problem and consistency. In Section 4 we derive an integral representation of additive cost sharing mechanisms which extends the isomorphism of Moulin and Shenker (1994) concerning the additive mechanisms with the constant returns property. Within the class of additive mechanisms the proportional mechanisms are the only consistent ones, assuming that positive demands always reward a positive share of total costs. Exchanging positivity by the weak fairness property equal treatment, we also end up with mechanisms billing in a proportional fashion. But now a preordering is used to preselect the real contributors as those with demands that are in its maximal indifference set. Egalitarian and average cost sharing belong to the mentioned class; the latter is further characterized by the property linearity, which weakens the standard constant returns. Adding positivity again, we obtain the class of quasi-proportional mechanisms.

In Section 5 is devoted to relaxations of the consistency property, weak consistency and regular consistency. The additive cost sharing mechanisms characterized by a consistent rationing method are regularly consistent, and, vice versa, each such cost sharing mechanism defines a consistent rationing method. In particular, the existence of these perfectly linked up notions of consistency provide a positive answer to the question raised in Moulin (2002, p. 328). We now may conclude that the serial mechanism is $R$-consistent, whereas the Shapley-Shubik mechanism is not. The characterization of parametric rationing methods of Young (1987) is extended to the context of cost sharing using this isomorphism. Finally we discuss converse consistency, the requirement that a solution for 2 agent problems be consistently extendible to problems with variable number of agents. 


\section{The MOdEL AND PRELIMINARIES}

Consider a production facility for some perfectly divisible good $Y$, of which the technology is summarized by a cost function $C: \mathbb{R}_{+} \rightarrow \mathbb{R}_{+} ; C(y)$ denotes the minimal (monetary) input to generate $y$ units of $Y$. It will be assumed that $C$ is nondecreasing and there are no fixed costs, so that $C(0)=0$. In addition we shall assume absolutely continuous cost functions ${ }^{1}$. This technical condition implies that a cost function is differentiable almost everywhere with respect to the Lebesgue measure $\lambda$. With slight abuse of notation $C^{\prime}$ is the marginal cost function, i.e., it coincides with the derivative of $C$ whenever the latter exists, and assumes the value 0 otherwise. In particular, $C^{\prime}$ is is $\lambda$-integrable and costs for output level $y$ may be expressed as $C(y)=\int_{0}^{y} C^{\prime}(t) \mathrm{d} t .^{2}$ The set of all cost functions is denoted by $\mathcal{C}$. Special cost functions are the base functions $B_{t}$ defined for $t \geq 0$ by $B_{t}(y)=\min \{t, y\}$, slant functions $\Lambda_{t}(y)=\max \{y-t, 0\}$ and the identity $C_{\text {id }}(y)=y$.

Let $\mathcal{N}$ be the set of nonempty and finite subsets of the natural numbers; $\mathcal{N}$ is interpreted as the set of potential agents in the cost sharing problem. A cost sharing problem for the set of agents $N \in \mathcal{N}$ is an ordered pair $P=(q, C) \in \mathbb{R}_{+}^{N} \times \mathcal{C}$. The interpretation of $P$ is that the agents in $N$ jointly own the production facility, and $q=\left(q_{i}\right)_{i \in N}$ summarizes the individual demands of the agents for good $Y$; then $q(N)=\sum_{i \in N} q_{i}$ is produced and cost $C(q(N))$ has to be shared. The set of all cost sharing problems for $N$ is denoted $\mathcal{P}^{N}$. The demand profile for $N$ containing only zeroes is denoted $\mathbf{0}_{N}$.

For $(q, c) \in \mathcal{P}^{N}, y \in \mathbb{R}_{+}^{N}$ is called vector of cost shares if $y(N)=C(q(N))$. A cost sharing mechanism assigns to each cost sharing problem a vector of cost shares. Reknown examples of mechanisms include average cost sharing, serial cost sharing (Moulin and Shenker (1992)), and the Shapley-Shubik cost sharing mechanism (Shubik (1962)). Concise definitions of these mechanisms are in the text below. The class of mechanisms with properties $P_{1}, \ldots, P_{m}$ will subsequently be denoted $M\left(P_{1}, \ldots, P_{m}\right)$.

\section{Contracts AND CONSISTENCY}

Assume that for each cost sharing problem $(q, C) \in \mathcal{P}^{N}$ not only it is specified how much an agent is billed but also how much he would consume and pay in case the actual production level were $y \leq q(N)$. A complete description for all contingent cost sharing problems will be called a contract. Formally, a contract for mechanism $\mu$ is an ordered pair $(\xi, \gamma)$ where for all cost

\footnotetext{
${ }^{1}$ For such functions it holds that for all intervals $[a, b] \subset \mathbb{R}_{+}$and $\varepsilon>0$ there is a $\delta>0$ such that for every finite collection of pairwise disjoint intervals $\left(a_{k}, b_{k}\right) \subset[a, b], k=1,2, \ldots, n$ with $\sum_{k=1}^{n}\left(b_{k}-a_{k}\right)<\delta$, we have $\sum_{k=1}^{n}\left|f\left(b_{k}\right)-f\left(a_{k}\right)\right|<\varepsilon$.

${ }^{2}$ This follows by the Fundamental Theorem in Lebesgue (1904).
} 
sharing problems $(q, C) \in \mathcal{P}^{N}$ the mapping $\xi(\cdot, q, C)$ describes the allocation of units available for consumption such that for all $y \in[0, q(N)]$

- $\sum_{i \in N} \xi_{i}(y, q, C)=y$, and

- $\xi(q(N), q, C)=q$.

The mapping $\gamma(\cdot, q, C)$ assigns to each production level $y$ vectors of cost shares such that

- $\sum_{i \in N} \gamma_{i}(y, q, C)=C(y)$, and

- $\gamma(q(N), q, C)=\mu(q, C)$.

I will make further assumptions. First, the mappings $y \mapsto \xi(y, q, C)$ and $y \mapsto \gamma(y, q, C)$ are non-decreasing. Second, for all $(q, C) \in \mathcal{P}^{N}, i \in N, k^{*}-\xi_{i}\left(k^{*}, q, C\right)=k^{\prime}-\xi_{i}\left(k^{\prime}, q, C\right)$ implies $C\left(k^{*}\right)-\gamma_{i}\left(k^{*}, q, C\right)=C\left(k^{\prime}\right)-\gamma_{i}\left(k^{\prime}, q, C\right)$. This condition expresses that if $i$ is the sole consumer of the last $k^{*}-k^{\prime}$ units then he pays for the corresponding incremental cost $C\left(k^{*}\right)-C\left(k^{\prime}\right)$.

Suppose that agent $i$ wants to leave group $N$. Then, as long as his demand is not met and his cost share not paid, agent $i$ leaves with a contract which stipulates that if a quantity $k \leq q(N)$ of the good is produced, he will receive a quantity $\xi_{i}(k, q, C)$ and pay a bill $\gamma_{i}(k, q, C)$. The agents in $N \backslash i$ are obliged to honor the very same contract and may use it to calculate the production cost of $y$ units for their own consumption as follows: first they find the level $k$ such that $y=k-\xi_{i}(k, q, C)$, then they produce $k$, give the quantity $\xi_{i}(k, q, C)$ to $i$, collect $\gamma_{i}(k, q, C)$ from him and end up paying the remainder of the cost, $C(k)-\gamma_{i}(k, q, C)$. This is taken as definition of the reduced cost $C_{(\xi, \gamma),-i}(y)$ for production level $y \leq q(N)$.

Definition 3.1 Let $\varphi=(\xi, \gamma)$ be a contract for mechanism $\mu$. For $(q, C) \in \mathcal{P}^{N}, i \in N$ the reduced cost function $C_{\varphi,-i}$ is defined by

$$
\begin{array}{ll}
y<q(N): \quad C_{\varphi,-i}(y)=C(k)-\gamma_{i}(k, q, C) \\
& \text { where } k \leq q(N) \text { solves } \sum_{j \in N \backslash i} \xi_{j}(k, q, C)=y, \\
y \geq q(N): \quad & C_{\varphi,-i}(y)=C(y)-\mu_{i}(q, C) .
\end{array}
$$

Note that $C_{\varphi,-i}$ indeed specifies an element in $\mathcal{C}$ : a solution to $\sum_{j \in N \backslash i} \xi_{j}(k, q, C)=y$ exists by continuity of $y \mapsto \xi(y, q, C)$. Moreover, $C_{\varphi,-i}$ is non-decreasing: $\sum_{i \in N} \gamma_{i}(y, q, C)=C(y)$ so for almost all $y, \sum_{i \in N} \frac{d}{d y} \gamma_{i}(y, q, C)=C^{\prime}(y)$, hence $\frac{d}{d y} \gamma_{i}(y, q, C) \leq C^{\prime}(y)$ by monotonicity.

Definition 3.2 Mechanism $\mu$ is consistent if for all contracts $\varphi$ for $\mu$, all $i \in N$, and all $(q, C) \in \mathcal{P}^{N}$

$$
\mu_{N \backslash i}(q, C)=\mu\left(q_{N \backslash i}, C_{\varphi,-i}\right) .
$$


Example 3.3 The average cost sharing mechanism $\mu^{\mathrm{AV}}$ defined for $q \neq \mathbf{0}_{N}$ by

$$
\mu^{\mathrm{AV}}(q, C)=q \cdot \frac{C(q(N))}{q(N)}
$$

is consistent (see Theorem 4.8) below. Now consider the serial mechanism $\mu^{\mathrm{SR}}$ introduced by Moulin and Shenker (1992). Take $(q, C) \in \mathcal{P}^{\{1,2, \ldots, n\}}$ and assume the demands are ordered such that $q_{i} \leq q_{j} \Leftrightarrow i \leq j$. Then with $x^{0}=0, x^{i}:=q_{1}+q_{2}+\ldots q_{i-1}+(n+1-i) q_{i}$ for all $i=1,2, \ldots, n$

$$
\mu_{i}^{\mathrm{SR}}(q, C)=\sum_{j=1}^{i} \frac{C\left(x^{j}\right)-C\left(x^{j-1}\right)}{n+1-j}
$$

Consider the contract $\varphi=(\xi, \gamma)$ defined by $\xi(y, q, C)=\frac{q}{q(N)} \cdot y, \gamma(y, q, C)=\frac{\mu^{\mathrm{sR}}(q, C)}{C(q(N))} C(y)$. Then for $(q, C) \in \mathcal{P}^{N}$ with $q=(1,2,3), C(y)=y^{2}, \mu^{\mathrm{SR}}(q, C)=(3,11,22)$ and $C_{\varphi,-1}(y)=\frac{33}{25} y^{2}$ so that $\mu_{\{2,3\}}\left(q_{\{2,3\}}, C_{\varphi,-1}\right)=\left(\frac{264}{25}, \frac{561}{25}\right) \neq \mu_{\{2,3\}}(q, C)$. So $\mu^{\mathrm{SR}}$ is not consistent.

\section{Consistent and AdDitive cost sharing}

As a first exercise I will study for the consistent members among the additive mechanisms, the class of mechanisms which accomodates most of the popular cost sharing mechanisms such as $\mu^{\mathrm{AV}}, \mu^{\mathrm{SR}}, \mu^{\mathrm{SS}}$.

\section{Additivity}

For all $C_{1}, C_{2} \in \mathcal{C}$, all $q \in \mathbb{R}_{+}^{N}, \mu\left(q, C_{1}+C_{2}\right)=\mu\left(q, C_{1}\right)+\mu\left(q, C_{2}\right)$.

Additivity is a standard property in the cost sharing literature which is usually motivated as an accounting convention. I will exploit the fact that the class of additive mechanisms allows for a transparent integral expression as in Moulin and Shenker (1994). ${ }^{3}$

\footnotetext{
${ }^{3}$ A precise statement in Moulin and Shenker (1994) is missing, so below I will refer to Moulin (2002), Th. 2.2 .
} 
4.1. Production assignment and rationing. A production sharing problem for agents in $N \in \mathcal{N}$ consists of a pair $(q, t) \in \mathbb{R}_{+}^{N} \times \mathbb{R}_{+}$such that $t \in[0, q(N)]$. Here $q$ is usually interpreted as a vector of claims, and $t$ the amount to be divided. A production assignment $\rho$ associates to each production sharing problem a vector $\rho(q, t) \in \mathbb{R}_{+}^{N}$ such that $\sum_{j \in N} \rho_{j}(q, t)=t$. Such $\rho$ is called rationing method if $\rho(q, t) \leq q$. In that case nobody is granted more than her claim while the available amount is fully distributed. The prevalent symmetric rationing methods are the proportional rationing method $r^{\mathrm{P}}$ and uniform gains method $r^{\mathrm{UG}}$. These are defined by $r^{\mathrm{P}}(q, t)=q / q(N) t$, and $r_{i}^{\mathrm{UG}}(q, t)=\min \left\{q_{i}, \omega\right\}$, where $\omega$ solves $\sum_{j \in N} \min \left\{q_{j}, \omega\right\}=t$. Consider a permutation $\sigma$ of $N$. Priority rationing relative to $\sigma$ is defined as $r_{i}^{\sigma}(q, t)=\max \{\min \{t-$ $\left.\left.\sum_{j: \sigma(j)<\sigma(i)} q_{j}, q_{i}\right\}, 0\right\}$. Random priority is defined by averaging the priority methods, $r^{\mathrm{RP}}(q, t)=$ $\frac{1}{n !} \sum_{\sigma} r^{\sigma}(q, t)$. See, e.g., Moulin (2002) and Thomson (2003) for overviews. ${ }^{4}$

Production assignment $\rho$ is monotonic whenever $t \leq t^{\prime}$ implies $\rho(q, t) \leq \rho\left(q, t^{\prime}\right)$ for all $t, t^{\prime}, q \in \mathbb{R}_{+}^{N}$; then $\rho$ defines for all $q \in \mathbb{R}_{+}^{N}$ a monotonic (and continuous) path $t \mapsto \rho(q, t)$ from 0 to $q$.

Any monotonic production assignment $\rho$ induces an additive cost sharing mechanism $\mu^{\rho}$ by

$$
\mu^{\rho}(q, C)=\int_{0}^{q(N)} C^{\prime}(t) \mathrm{d} \rho(q, t) .
$$

We may also reverse the statement, since each additive mechanism may be expressed in this way by choosing a suitable production assignment :

Theorem 4.1 $\mu \in M(A D D)$ if and only if $\mu=\mu^{\rho}$ for some monotonic production assignment

$\rho$.

Proof. The 'if' part is clear, which leaves us to show the 'only if' part. Take $\mu \in M(A D D)$. Define a production assignment $\rho$ by $\rho(q, t):=\mu\left(q, B_{t}\right)$. Then it is easy to see that $\rho$ is monotonic. Take $q \in \mathbb{R}_{+}^{N}$ and $x, y \in[0, q(N)], x \leq y$. Then $B_{y}-B_{x} \in \mathcal{C}$ and

$$
\rho(q, y)-\rho(q, x)=\mu\left(q, B_{y}\right)-\mu\left(q, B_{x}\right)=\mu\left(q, B_{y}-B_{x}\right) \geq 0 .
$$

Now consider the function $\Lambda_{x}(y):=B_{q(N)}(y)-B_{x}(y)=\max \{y-x, 0\}$. Then

$$
\begin{aligned}
\mu^{\rho}\left(q, \Lambda_{x}\right) & =\int_{0}^{q(N)} \Lambda_{x}^{\prime}(t) d \rho(q, t)=\int_{x}^{q(N)} 1 d \rho(q, t)=\rho(q, q(N))-\rho(q, x) \\
& =\mu\left(q, B_{q(N)}\right)-\mu\left(q, B_{x}\right)=\mu\left(q, B_{q(N)}-B_{x}\right)=\mu\left(q, \Lambda_{x}\right) .
\end{aligned}
$$

Then we may conclude that $\mu=\mu^{\rho}$ by Lemma 1 in Moulin and Shenker (1994).

\section{Constant returns}

For any $\alpha \geq 0, \mu\left(q, \alpha C_{\text {id }}\right)=\alpha q$.

\footnotetext{
${ }^{4}$ Moulin (2000) focuses on discrete formulation of the problem and asymmetric priority rules.
} 
Corollary 4.2 (Moulin and Shenker (1994)) $\mu \in M(A D D, C R) \Leftrightarrow \mu=\mu^{r}$ for some monotonic rationing method $r$.

Equal Treatment $q_{i}=q_{j}$ implies $\mu_{i}(q, C)=\mu_{j}(q, C)$.

Similarly, any production assignment $\rho$ satisfies equal treatment if for all production sharing problems $(q, t), q_{i}=q_{j}$ implies $\rho_{i}(q, t)=\rho_{j}(q, t)$.

Theorem $4.3 \mu \in M(\mathrm{ADD}, \mathrm{ET})$ if and only if $\mu=\mu^{\rho}$ and $\rho$ satisfies $\mathrm{ET}$.

Proof. We only show the 'only if' part. ADD implies the functional representation as in Theorem 4.1. Now suppose there is $q \in \mathbb{R}_{+}^{N}$ such that $q_{i}=q_{j}$ but not $\rho_{i}(q, t)=\rho_{j}(q, t)$ for all $t \in[0, q(N)]$. Assume that not $\rho_{i}(q, t) \geq \rho_{j}(q, t)$ for all $t \in[0, q(N)]$. Then there must be an interval $U$ such that $\frac{d}{d t} \rho_{i}(q, t)<\frac{d}{d t} \rho_{j}(q, t)$ for all $t \in U$. Consider the indicator function $\mathbb{I}_{U}$ with respect to $U$ and define $C \in \mathcal{C}$ by $C(y)=\int_{0}^{y} \mathbb{I}_{U}(t) \mathrm{d} t$ for all $y \in \mathbb{R}_{+}$. Then $\mu_{i}(q, C)=\int_{0}^{q(N)} \mathbb{I}_{U}(t) \mathrm{d} \rho_{i}(q, t)<\int_{0}^{q(N)} \mathbb{I}_{U}(t) \mathrm{d} \rho_{j}(q, t)=\mu_{j}(q, C)$, contradicting ET.

Within the demand sharing context the idea of consistency is transparent and intuitive: a production assignment $\rho$ is called consistent if for all demand sharing problems $(q, x)$ among agents in $N$, all $j \in N$,

$$
\rho_{N \backslash j}(q, x)=\rho\left(q_{N \backslash j}, x-\rho_{j}(q, x)\right) .^{5}
$$

Hence, consistency states that with removing an agent from the cooperative $N$, and taking all the resources that are allocated to this agent, renewed allocation of the remaining pieces within the reduced society does not make a difference as long as $\rho$ is used. As Moulin (2000) puts it within the context of rationing, 'changing the status of an agent from active participant to passive expense of resources does not alter the overall distribution'.

Proposition 4.4 If $\mu$ is additive and consistent then $\mu=\mu^{\rho}$ with consistent and monotonic $\rho$.

Proof. If $\mu$ is additive then take $\rho$ as is guaranteed by Theorem 4.1. Define the contract $\varphi=(\xi, \gamma)$ by $\xi(y, q, C)=\rho(q, y)$ and $\gamma(y, q, C)=\int_{0}^{y} C^{\prime}(t) \mathrm{d} \rho(q, t)$. Then for $x \in[0, q(N)], y \in[0, x]$ we have $\xi\left(y, q, B_{x}\right)=\rho(q, y)=\gamma\left(y, q, B_{x}\right)$. Hence, for $i \in N,\left(B_{x}\right)_{\varphi,-i}=B_{x-\rho_{i}(q, x)}$, and thus, by

\footnotetext{
${ }^{5}$ In fact the notion is usually defined in terms of general sets of agents leaving, but is derived from repeated application of this statement.
} 
consistency

$$
\begin{aligned}
\rho_{N \backslash i}(q, x) & =\mu_{N \backslash i}\left(q, B_{x}\right)=\mu\left(q_{N \backslash i},\left(B_{x}\right)_{\varphi,-i}\right)=\int_{0}^{q(N \backslash i)}\left(B_{x-\rho_{i}(q, x)}\right)^{\prime}(t) \mathrm{d} \rho\left(q_{N \backslash i}, t\right) \\
& =\int_{0}^{x-\rho_{i}(q, x)} \mathrm{d} \rho\left(q_{N \backslash i}, t\right)=\rho\left(q_{N \backslash i}, x-\rho_{i}(q, x)\right) .
\end{aligned}
$$

Example 4.5 The converse does not hold: $r^{\mathrm{UG}}$ defines a consistent production assignment but $\mu^{\mathrm{SR}}=\mu^{r^{\mathrm{VG}}}$ is not consistent.

Example 4.6 $\mu^{\mathrm{SS}}$ is not consistent since $\mu^{\mathrm{SS}}=\mu^{r^{\mathrm{RP}}}$ and $r^{\mathrm{RP}}$ is not a consistent rationing method.

Null Agent If $q_{i}=0$ then $\mu_{i}(q, C)=0$.

Lemma 4.7 If $\mu \in M(\mathrm{CONS})$ then $\mu \in M$ (NULL).

Proof. Let $\varphi$ be a contract for $\mu \in M(\mathrm{CONS})$ and take a cost sharing problem $P=(q, C) \in \mathcal{P}^{N}$ with $q_{i}=0$ for some agent $i \in N$. Denote $N \backslash i=\left\{i_{1}, i_{2}, i_{3}, \ldots, i_{n-1}\right\}$. Define iteratively cost sharing problems $P^{k}=\left(q^{k}, C^{k}\right)$ for the agents $N^{k}=\left\{i, i_{k}, i_{k+1}, \ldots, i_{n-1}\right\}$ for $k=1,2, \ldots, n-1$ so that $P^{1}=P$ and $q^{k}:=q_{i, i_{k}, i_{k+1}, \ldots, i_{n-1}}$ and $C^{k}=\left(C^{k-1}\right)_{\varphi,-i_{k-1}}$ for $k \geq 2$. By consistency we have

$$
\mu_{i}(P)=\mu_{i}\left(P^{1}\right)=\ldots=\mu_{i}\left(P^{n-1}\right)=\mu_{i}\left(q_{i}, C^{n-1}\right) .
$$

Now, since $C^{n-1} \in \mathcal{C}$, we obtain $C^{n-1}(0)=0$ which in turn implies $\mu_{i}\left(q_{i}, C^{n-1}\right)=0$.

\section{Positive cost for positive demand}

For any $C \in \mathcal{C}$, any $i \in N$, and any $q \in \mathbb{R}_{+}^{N}$, if $q_{i}>0$ while $C(q(N))>0$ then $\mu_{i}(q, C)>0$.

Theorem 4.8 Assume $n \geq 3$. Mechanism $\mu$ satisfies additivity, consistency, and positive cost for positive demand if and only if for each $i \in N$ there is a function $f_{i}: \mathbb{R}_{+} \rightarrow \mathbb{R}_{+}$such that $f_{i}(y)=0 \Leftrightarrow y=0$ and for all nontrivial $(q, C) \in \mathcal{P}^{N}$

$$
\mu_{i}(q, C)=\frac{f_{i}\left(q_{i}\right)}{\sum_{j \in N} f_{j}\left(q_{j}\right)} C(q(N)) .
$$


Proof. First we show the implication $\Leftarrow$. Suppose $\mu$ takes the form as in (4). Consider the contract $(\xi, \gamma)$ for $\mu$ defined for $(q, C) \in \mathcal{P}^{N}$ by

$$
\xi_{i}(y, q, C)=\frac{q_{i}}{q(N)} y, \gamma_{i}(y, q, C)=\frac{f_{i}\left(q_{i}\right)}{\sum_{k \in N} f_{j}\left(q_{j}\right)} C(y) .
$$

Take $i \in N$, then for each $y \geq 0$ we obtain

$$
k-\xi_{i}(k, q, C)=y \Longrightarrow k=\frac{q(N)}{q(N \backslash i)} y .
$$

Then

$$
\begin{aligned}
C_{(\xi, \gamma),-i}(y) & =C\left(\frac{q(N)}{q(N \backslash i)} y\right)-\gamma_{i}\left(\frac{q(N)}{q(N \backslash i)} y, q, C\right) \\
& =C\left(\frac{q(N)}{q(N \backslash i)} y\right)-\frac{f_{j}\left(q_{j}\right)}{\sum_{k \in N} f_{k}\left(q_{k}\right)} C\left(\frac{q(N)}{q(N \backslash i)} y\right) \\
& =\frac{\sum_{j \in N \backslash i} f_{j}\left(q_{j}\right)}{\sum_{j \in N} f_{j}\left(q_{j}\right)} C\left(\frac{q(N)}{q(N \backslash i)} y\right) .
\end{aligned}
$$

Hence for $j \in N \backslash i$ :

$$
\mu_{j}\left(q_{N \backslash i}, C_{(\xi, \gamma),-i}\right)=\frac{f_{j}\left(q_{j}\right)}{\sum_{k \in N \backslash i} f_{i}\left(q_{k}\right)} C_{(\xi, \gamma),-i}(q(N \backslash i))=\frac{f_{j}\left(q_{j}\right)}{\sum_{k \in N} f_{k}\left(q_{k}\right)} C(q(N))=\mu_{j}(q, C) .
$$

' $\Rightarrow$ ' Assume $\mu \in M(\mathrm{ADD}, \mathrm{CONS})$. By Theorem 4.1 there is $\rho$ such that $\mu=\mu^{\rho}$. Define the following contract for $\mu, \xi(y, q, C)=\frac{q}{q(N)} y, \gamma(y, q, C)=\frac{\rho(q, q(N))}{c(q(N))} C(y)$ for $(q, C) \in \mathcal{P}^{N}$ such that $q(N)>0$. Now take $(q, C) \in \mathcal{P}^{N}$, and assume without loss of generality that $q_{i}>0, q(N \backslash i)>0$. Consistency implies

$$
C_{\varphi,-i}(y)=\frac{\sum_{j \in N \backslash i} \rho_{j}(q, q(N))}{q(N)} C\left(\frac{q(N)}{q(N \backslash i)} y\right)
$$

In particular,

$$
\begin{aligned}
\left(B_{t}\right)_{\varphi,-i}(y) & =\frac{\sum_{j \in N \backslash i} \rho_{j}(q, q(N))}{q(N)} B_{t}\left(\frac{q(N)}{q(N \backslash i)} y\right)= \\
& =\frac{\sum_{j \in N \backslash i} \rho_{j}(q, q(N))}{q(N)} \cdot \frac{q(N)}{q(N \backslash i)} B_{\frac{q(N \backslash i)}{q(N)} t}(y)= \\
& =\frac{\sum_{j \in N \backslash i} \rho_{j}(q, q(N))}{q(N \backslash i)} \cdot B_{\frac{q(N \backslash i)}{q(N)} t}(y) .
\end{aligned}
$$


Again by consistency and additivity

$$
\begin{aligned}
\rho_{N \backslash i}(q, t) & =\mu_{N \backslash i}^{\rho}\left(q, B_{t}\right)=\mu^{\rho}\left(q_{N \backslash i},\left(B_{t}\right)_{\varphi,-i}\right)= \\
& =\frac{\sum_{j \in N \backslash i} \rho_{j}(q, q(N))}{q(N \backslash i)} \cdot \mu^{\rho}\left(q_{N \backslash i}, B_{\frac{q(N \backslash i)}{q(N)} t}(y)\right)= \\
& =\frac{\sum_{j \in N \backslash i} \rho_{j}(q, q(N))}{q(N \backslash i)} \cdot \rho\left(q_{N \backslash i}, \frac{q(N \backslash i)}{q(N)} t\right) .
\end{aligned}
$$

This equality has two consequences:

(A) By taking sums on the left and right we get, for $t \in[0, q(N)]$

$$
\begin{aligned}
t-\rho_{i}(q, t) & =\sum_{k \in N \backslash i} \rho_{k}(q, t)=\frac{q(N)-\rho_{i}(q, q(N))}{q(N \backslash i)} \cdot \sum_{k \in N \backslash i} \rho_{k}\left(q_{N \backslash i}, \frac{q(N \backslash i)}{q(N)} t\right)= \\
& =\frac{q(N)-\rho_{i}(q, q(N))}{q(N \backslash i)} \cdot \frac{q(N \backslash i)}{q(N)} t=t-\frac{\rho_{i}(q, q(N))}{q(N)} t .
\end{aligned}
$$

So for $t \in[0, q(N)]$,

$$
\rho_{i}(q, t)=\frac{\rho_{i}(q, q(N))}{q(N)} t .
$$

Hence

$$
\mu^{\rho}(q, C)=\frac{\rho(q, q(N))}{q(N)} C(q(N))
$$

(B) For $q \gg 0$, we get by substituting $t=q(N)$ for all $j, k \neq i$ and positive cost shares for positive demands that

$$
\frac{\rho_{k}(q, q(N))}{\rho_{j}(q, q(N))}=\frac{\rho_{k}\left(q_{N \backslash i}, q(N \backslash i)\right)}{\rho_{j}\left(q_{N \backslash i}, q(N \backslash i)\right)} .
$$

So the left-hand fraction is actually independent from the value $q_{i}$. By varying over all triples $i, j, k$, the conclusion is that the following expression is well-defined

$$
h_{i j}\left(q_{i}, q_{j}\right):=\frac{\rho_{i}(q, q(N))}{\rho_{j}(q, q(N))} \text { for all } i, j \in N .
$$

Then

$$
h_{i j}\left(q_{i}, q_{j}\right) h_{j k}\left(q_{j}, q_{k}\right) h_{k i}\left(q_{k}, q_{i}\right)=1 \text { for different } i, j, k \in N,
$$

and, in particular, we find that any expression of type $h_{j k}\left(q_{j}, q_{k}\right) h_{k i}\left(q_{k}, q_{i}\right)$ is independent from $q_{k}$. I claim that there are functions $f_{j}: \mathbb{R} \backslash\{0\} \rightarrow \mathbb{R}_{+}$such that

$$
h_{i j}\left(q_{i}, q_{j}\right)=\frac{f_{i}\left(q_{i}\right)}{f_{j}\left(q_{j}\right)} .
$$


To see this, fix $k \in N$ and define, invoking the independence resulting from (10),

$$
\begin{aligned}
f_{i}\left(q_{i}\right): & =h_{k i}\left(1, q_{i}\right) \text { for all } i \neq k, \\
f_{k}\left(q_{k}\right): & =h_{k \ell}(1, y) h_{k \ell}\left(q_{k}, y\right) \text { for some } \ell \neq k, y \neq 0 .
\end{aligned}
$$

Then, finally, we are able to assert our claim since for $i, j \neq k$ :

$$
\frac{f_{i}\left(q_{i}\right)}{f_{j}\left(q_{j}\right)}=\frac{h_{k i}\left(1, q_{i}\right)}{h_{k j}\left(1, q_{j}\right)}=\frac{1}{h_{i k}\left(q_{i}, 1\right) h_{k j}\left(1, q_{j}\right)}=h_{i j}\left(q_{i}, q_{j}\right)
$$

and by choosing $\ell=i, y=q_{i}$ in (11):

$$
\frac{f_{i}\left(q_{i}\right)}{f_{k}\left(q_{k}\right)}=\frac{h_{k i}\left(1, q_{i}\right)}{h_{k i}\left(1, q_{i}\right) h_{k i}\left(q_{k}, q_{i}\right)}=h_{k i}\left(q_{k}, q_{i}\right) .
$$

So I established the claim, as for all $i, j \in N$

$$
\frac{\rho_{j}(q, q(N))}{\rho_{i}(q, q(N))}=\frac{f_{j}\left(q_{j}\right)}{f_{i}\left(q_{i}\right)} .
$$

It is not hard to see that (12) is true for any $q \in \mathbb{R}_{+}^{N}$ such that $q_{i}>0$ and all $j \in N$. Proof by induction on the number of zero demanders in $q, Z(q)$. Suppose the statement is correct for all $q$ with $q_{i}>0$ and $Z(q) \leq k-1$ for $1 \leq k \leq n-1$. Now take $q$ with $q_{i}>0, Z(q)=k$. For $h$ with $q_{h}=0$ and $j \neq h, \epsilon>0$

$$
\frac{\rho_{j}(q, q(N))}{\rho_{i}(q, q(N))}=\frac{\rho_{j}\left(q_{N \backslash h}, q(N \backslash h)\right)}{\rho_{i}\left(q_{N \backslash h}, q(N \backslash h)\right)}=\frac{\rho_{k}\left(\left(q_{N \backslash h}, \epsilon\right), q(N \backslash h)+\epsilon\right)}{\rho_{j}\left(\left(q_{N \backslash h}, \epsilon\right), q(N \backslash h)+\epsilon\right)}=\frac{f_{j}\left(q_{j}\right)}{f_{i}\left(q_{i}\right)} .
$$

Here, the last equality is due to the induction hypothesis.

Now, by combining (A) and (B) we are finally done since for all $q_{i}>0$

$$
\begin{aligned}
\mu_{i}^{\rho}(q, C) & =\frac{\rho_{i}(q, q(N))}{q(N)} C(q(N))=\frac{\rho_{i}(q, q(N))}{\sum_{j \in N} \rho_{j}(q, q(N))} C(q(N)) \\
& =\frac{\rho_{i}(q, q(N))}{\frac{\rho_{i}(q, q(N))}{f_{i}\left(q_{i}\right)} \sum_{j \in N} f_{j}\left(q_{j}\right)} C(q(N))=\frac{f_{i}\left(q_{i}\right)}{\sum_{j \in N} f_{j}\left(q_{j}\right)} C(q(N)) .
\end{aligned}
$$

Well-known examples in the class of all such mechanisms are the proportional rule with $f(y)=y$, the egalitarian rule with $f(y)=1$ whenever $y>0$. A few examples that have become classic in queuing theory and network performance evaluation are limited (discrimanatory) processor sharing $f_{i}(y)=w_{i} \min \left\{y, \ell_{i}\right\}$ and coupled processor allocation $f_{i}(y)=\ell_{i} \mathbb{I}_{\mathbb{R}_{++}}(y)$.

Remark At first glance consistency may seem a very strong notion, as a solution should offer stability with respect to varying sets of agents relative to the unrestricted domain of contracts. However, note that in Theorem 4.8 only very special contracts are used to arrive at the formula (8). 
The following examples point out that the three axioms additivity, consistency and positive cost for positive demand are logical independent.

Example 4.9 Define the following mechanism $\mu$. Select two agents $i, j \in N$. Define, for any $C \in \mathcal{C}$, any $q \in \mathbb{R}_{+}^{N}, \mu(q, C)=\mu^{\mathrm{AV}}(q, C)$ if $\left\{k \in N \mid q_{k}=0\right\}=\{i, j\}$, and else $\mu_{\{i, j\}}(q, C):=$ $(0,0), \mu_{N \backslash\{i, j\}}(q, C)=\frac{q_{N \backslash\{i, j\}}}{q(N \backslash\{i, j\})} C(q(N))$. Then $\mu$ is additive and consistent, but fails to charge the agents $i, j$ a positive share of positive costs.

Example 4.10 For any $(q, C) \in \mathcal{P}^{N}, j \in N$ define $f_{j}(q, C)=C\left(q(N)+q_{j}\right)-C(q(N))+q_{i}$. Define a cost sharing mechanism $\mu$ by

$$
\mu_{i}(q, C)=\frac{f_{i}(q, C)}{\sum_{j \in N} f_{j}(q, C)} C(q(N)) .
$$

Then $\mu$ is consistent, since any reduction leaves the values of $f_{j}$ unchanged. Moreover, since $f_{i}(q, C)=0$ if and only if $q_{i}=0, \mu$ satisfies positive cost for positive demand. But clearly $\mu$ is not additive.

Example 4.11 Below we will see that the Shapley-Shubik mechanism fails to be weakly consistent, and therefore it does not constitute a consistent solution. But the mechanism is additive and does satisfy positive cost for positive demand.

Adding ET to the characterization in Theorem 4.8 leads to a characterization of the family of quasi-proportional mechanisms: ${ }^{6}$

Corollary 4.12 Assume $n \geq 3$. Then $\mu \in M(\mathrm{ADD}, \mathrm{CONS}, \mathrm{POS}, \mathrm{ET})$ if and only if there is a function $f: \mathbb{R}_{+} \rightarrow \mathbb{R}_{+}$with $f(x)=0 \Leftrightarrow x=0$ such that

$$
\mu_{i}(q, C)=\frac{f\left(q_{i}\right)}{\sum_{j \in N} f\left(q_{j}\right)} C(q(N)) \text { for all } i \in N,(q, C) \in \mathcal{P}^{N}, q \neq \mathbf{0} .
$$

Proof. Take $\mu \in M(\mathrm{ADD}, \mathrm{CONS}, \mathrm{POS}, \mathrm{ET}),(q, C) \in \mathcal{P}^{N}$ and functions $\left\{f_{j}\right\}_{j \in N}$ as in Theorem 4.8. Then by ET, for arbitrary $i, j \in N, q_{i}=q_{j} \Longrightarrow f_{i}\left(q_{i}\right)=f_{j}\left(q_{j}\right)$ and so we obtain $f_{i}=f_{j}$ for all $i, j$.

\footnotetext{
${ }^{6}$ The name is borrowed from Moulin and Sprumont (2005) who discuss the same family, only for a discrete framework.
} 
Example 4.13 Positivity can not be left out. Define $\mu_{i}(q, C)$ for $(q, C) \in \mathcal{P}^{N}$ as follows. If $q_{i} \leq 1$ for all $i \in N$, then define $\mu(q, C)=\mu^{\mathrm{AV}}(q, C)$, else let $S=\left\{i \in N \mid q_{i}>1\right\}$ and put $\mu_{N \backslash S}(q, C)=0$ and $\mu_{S}(q, C)=\frac{q_{S}}{q(S)} C(q(N))$. Then $\mu$ satisfies all but the property POS. $\triangleleft$

For a given preordering $\preccurlyeq$ of $\mathbb{R}_{+}$, any $q \in \mathbb{R}_{+}^{N}$, denote by $A(q, \preccurlyeq) \subseteq N$ the set of agents for which the demands in $q$ are in the maximal indifference set of $\preccurlyeq$, i.e., $i \in A(q, \preccurlyeq)$ if there is no $j \in N$ such that $q_{i} \prec q_{j}$.

Theorem 4.14 Assume $n \geq 3$. Then $\mu \in M(\mathrm{ADD}, \mathrm{CONS}, \mathrm{ET})$ if and only if there is a preordering $\preccurlyeq$ of $\mathbb{R}_{+}$and a function $f: \mathbb{R}_{+} \rightarrow \mathbb{R}_{+}$such that for each $q \in \mathbb{R}_{+}^{N}, C \in \mathcal{C}$

$$
\mu_{i}(q, C)= \begin{cases}\frac{f\left(q_{i}\right)}{\sum_{j \in A(q, \preccurlyeq)} f\left(q_{j}\right)} C(q(N)) & \text { if } i \in A(q, \preccurlyeq), \\ 0 & \text { if } i \notin A(q, \preccurlyeq) .\end{cases}
$$

Proof. We will only show the 'only if' part. Let $\mu \in M$ (ADD, CONS, ET) and take a production assignment $\rho$ such that $\mu=\mu^{\rho}$ as is guaranteed by Theorem 4.1. Below I will use (5) for 3agent sets $U \in \mathcal{N}$ : for any $q \in \mathbb{R}_{+}^{U}$, and all pairs $u, v \in U$ we have $\rho_{\{u, v\}}(q, q(U))=0_{\{u, v\}}$ if $q_{\{u, v\}}=0_{\{u, v\}}$, and

$$
\rho_{\{u, v\}}(q, q(U))=\frac{\rho_{u}(q, q(U))+\rho_{v}(q, q(U))}{q_{u}+q_{v}} \rho\left(q_{\{u, v\}}, q_{u}+q_{v}\right) .
$$

otherwise.

Define a complete ordering $\preccurlyeq$ on $\mathbb{R}_{+}$by

$$
q_{i} \preccurlyeq q_{j} \text { if } \rho\left(\left(q_{i}, q_{j}\right), q_{i}+q_{j}\right) \gg 0 \text { or } \rho_{i}\left(\left(q_{i}, q_{j}\right), q_{i}+q_{j}\right)=0 .
$$

Claim: $\preccurlyeq$ defines a preordering.

$1) \preccurlyeq$ is reflexive, i.e., $q_{i} \preccurlyeq q_{i}$ for all $q_{i} \in \mathbb{R}_{+}$. By ET it holds $\rho_{i}\left(\left(q_{i}, q_{i}\right), 2 q_{i}\right)=q_{i}$, hence if $q_{i}=0$ then $\rho_{i}\left(\left(q_{i}, q_{i}\right), 2 q_{i}\right)=0$ and $\rho_{i}\left(\left(q_{i}, q_{i}\right), 2 q_{i}\right)=\left(q_{i}, q_{i}\right) \gg 0$ otherwise.

$2) \preccurlyeq$ is transitive, i.e., if $q_{i} \preccurlyeq q_{j}$ and $q_{j} \preccurlyeq q_{k}$, then $q_{i} \preccurlyeq q_{k}$.

Take $q_{i}, q_{j}, q_{k} \in \mathbb{R}_{+}$and assume $q_{i} \preccurlyeq q_{j}$ and $q_{j} \preccurlyeq q_{k}, q_{i} \succ q_{k}$. Then $q_{i}>0$ by NULL and $\rho_{k}\left(q_{\{i, k\}}, q_{i}+q_{k}\right)=0$. Now apply (15) to $\{u, v\}=\{i, k\}$ and conclude that $\rho_{k}(q, q(U))=0$.

Distinguish between the two cases:

(A) $q_{j} \prec q_{k}$. Then $\rho_{j}\left(q_{\{j, k\}}, q_{j}+q_{k}\right)=0$, hence by (15) with $\{u, v\}=\{j, k\}, \rho_{j}(q, q(U))=0$. Then $\rho_{i}(q, q(U))=q(U)>0$. Now by (15) for $\{u, v\}=\{i, j\}, \rho_{j}\left(q_{\{i, j\}}, q_{i}+q_{j}\right)=0$ and $\rho_{i}\left(q_{\{i, j\}}, q_{i}+q_{j}\right)>0$, contradicting $q_{i} \preccurlyeq q_{j}$.

(B) $q_{j} \sim q_{k}$. Without loss of generality assume $q_{j} \neq q_{k}$. Then $\rho\left(q_{\{j, k\}}, q_{j}+q_{k}\right) \gg 0$ and by application of (15) to $\{u, v\}=\{j, k\}$ we obtain $\rho_{j}(q, q(N))+\rho_{k}(q, q(U))=0$, so $\rho_{j}(q, q(N))=0$. Then, like in case (A), we obtain $\rho_{i}(q, q(U))=q(U)>0$, which leads to contradiction with 
$q_{i} \preccurlyeq q_{j}$. In both the cases (A) and (B) the assumption $q_{k} \prec q_{i}$ leads to a contradiction, so $q_{i} \preccurlyeq q_{k}$. This proves transitivity.

Take $N \in \mathcal{N}, q \in \mathbb{R}_{+}^{N}$ and suppose for $i, j \in N$ that $q_{i} \prec q_{j}$. Let $N \backslash\{i, j\}=\left\{k_{1}, k_{2}, \ldots, k_{n-2}\right\}$ and define $T_{0}=\varnothing, T_{\ell}=\left\{k_{1}, k_{2}, \ldots, k_{\ell}\right\}$. Then by successive removal of the agents $k_{1}, k_{2}, \ldots, k_{n-2}$ by CONS and (5),

$$
\rho_{\{i, j\}}(q, q(N))=\prod_{\ell=0}^{n-2} \sum_{t \in N \backslash T_{\ell}} \frac{\rho_{t}\left(q_{N \backslash T_{\ell}}, q\left(N \backslash T_{\ell}\right)\right)}{q\left(N \backslash T_{\ell}\right)} \cdot \rho\left(q_{\{i, j\}}, q_{i}+q_{j}\right),
$$

which shows that $\rho_{i}(q, q(N))=0$. In particular, the only agents making positive contributions are those with demands in the maximal indifference class of $\preccurlyeq$. If $q \neq \mathbf{0}_{N}$, the only interesting case, then $A(q, \preccurlyeq) \cap A\left(\mathbf{0}_{N}, \preccurlyeq\right)=\varnothing$ which means that for all $i \in A(q, \preccurlyeq), \rho_{i}(q, q(N))>0$. Then this suffices to be able to repeat the reasoning as in the second part of the proof of Theorem 4.8. The same logic applies to derive values $f_{i}\left(q_{i}\right)$ for agent $i \in A(q, \preccurlyeq)$, and by ET the functions $f_{i}$ are all the same so that we may put $f\left(q_{i}\right)=f_{i}\left(q_{i}\right)$ for all $i \in A(q, \preccurlyeq)$. Then basically this shows how to derive the values of the desired function $f$ on the maximal indifference class corresponding to $q$. We only need to be cautious where the induction hypothesis is applied: $\epsilon$ can not be any positive value, but needs to be in the maximal indifference class determined by $q$. Then finally, complete the definition of $f$ by varying $q$ so that $f$ gets values on all the indifference classes.

\section{Linearity}

For all $\alpha, \beta \in \mathbb{R}_{+}$, it holds $\mu_{i}\left(\left(q_{-i}, \beta q_{i}\right), \alpha C_{\text {id }}\right)=\beta \mu_{i}\left(q, \alpha C_{\text {id }}\right)$.

This property is similar to that discussed in Trudeau (2009). In absence of externalities it imposes that a raise or decrease of ones individual demand with a factor $\beta$ will magnify the bill in the cost sharing problem accordingly. Notice that it is weaker than the constant returns property.

Theorem 4.15 Assume $n \geq 3$. Then $\mu \in M(\mathrm{ADD}, \mathrm{CONS}, \mathrm{LIN}, \mathrm{ET})$ if and only if $\mu=\mu^{\mathrm{AV}}$.

Proof. We only show the 'only if' part. Suppose that $\mu \in M$ (ADD, CONS, LIN, ET). We have $\mu=\mu^{\rho}$ and by (8):

$$
\mu^{\rho}(q, C)=\frac{\rho(q, q(N))}{q(N)} C(q(N)) \text { for all }(q, C) \in \mathcal{P}^{N} \text { with } q(N)>0 .
$$

Now fix $N=\{1,2, \ldots, n\}, q \in \mathbb{R}_{+}^{N}$. If $q(N)=0$ then by nonnegativity of cost shares $\mu(q, C)=$ $\mathbf{0}_{N}=\mu^{\mathrm{AV}}(q, C)$. So we may assume $q \neq \mathbf{0}_{N}$ and, without loss of generality, that $q_{1}>0$. We 
will show that $\rho(q, q(N))=q$ for all $q_{N \backslash 1} \in \mathbb{R}_{+}^{N \backslash 1}$ by (increasing) induction on the number of agents with demands unequal to $q_{1}$, i.e. the size of $S(q)=\left\{j \in N \backslash 1 \mid q_{j} \neq q_{1}\right\}$. First of all, if $|S(q)|=0$, then ET implies $\rho(q, q(N))=q$. Now suppose $\rho(q, q(N))=q$ if $|S(q)| \leq k$ for some $k \leq n-1$. Take a demand profile $q$ with $|S(q)|=k+1$. For $i \in S(q)$, by application of linearity and the induction hypothesis

$$
\rho_{i}(q, q(N))=\rho_{i}\left(\left(q_{-i}, \frac{q_{i}}{q_{1}} \cdot q_{1}\right), q(N)\right)=\frac{q_{i}}{q_{1}} \cdot \rho_{i}\left(\left(q_{-i}, q_{1}\right), \sum_{j \neq i} q_{j}+q_{1}\right)=\frac{q_{i}}{q_{1}} \cdot q_{1}=q_{i} .
$$

In addition, by ET it holds for all $i \notin S(q)$

$$
\rho_{i}(q, q(N))=\frac{q(N)-\sum_{j \in S(q)} \rho_{j}(q, q(N))}{|N \backslash S(q)|}=\frac{|N \backslash S(q)| q_{1}}{|N \backslash S(q)|}=q_{1} .
$$

Then we have shown that $\rho(q, q(N))=q$.

Example 4.16 $\mu^{\mathrm{E}}$ satisfies all of the above properties except LIN.

\section{Relaxing CONSISTEnCy, COnsistent Rationing}

Consistency stipulates that a mechanism should be able to take care of all kinds of contracts - even the less sensible ones. Then this property seems overly strong when the agents are willing to accept any contract in agreement with the idea of preserving the cost shares in the induced reduced cost sharing problems.

Definition 5.1 Mechanism $\mu$ is weakly consistent if a contract $\varphi$ for $\mu$ exists, such that for all $i \in N$, and all $(q, C) \in \mathcal{P}^{N}, \mu_{N \backslash i}(q, C)=\mu\left(q_{N \backslash i}, C_{\varphi,-i}\right)$.

Theorem 5.2 If $\mu=\mu^{\rho}$ and $\rho$ is a consistent production assignment, then $\mu$ is weakly consistent.

Proof. Assume $\rho$ is a consistent and monotonic production assignment and $\mu=\mu^{\rho}$. Define the contract $\varphi=(\xi, \gamma)$ for $\mu$ by $\xi(y, q, C)=\rho(q, y)$ and $\gamma(y, q, C)=\int_{0}^{y} C^{\prime}(t) \mathrm{d} \rho(q, t)$. For $i \in N, y \in[0, q(N)]$

$$
\begin{aligned}
C_{\varphi,-i}\left(y-\rho_{i}(q, y)\right) & =C_{\varphi,-i}\left(y-\xi_{i}(y, q, C)\right) \\
& =C(k)-\gamma_{i}(k, q, C)=\int_{0}^{k}\left(1-\frac{d}{d t} \rho_{i}(q, t)\right) C^{\prime}(t) \mathrm{d} t .
\end{aligned}
$$


where $k$ is such that $y=k-\xi_{i}(k, q, C)=k-\rho_{i}(q, k)$. Substituting the latter expression for $y$ and using the chain rule we obtain for almost all $y \in[0, q(N)]^{7}$

$$
\left(1-\frac{d}{d y} \rho_{i}(q, y)\right)\left(C_{(\xi, \gamma),-i}\right)^{\prime}\left(y-\rho_{i}(q, y)\right)=\left(1-\frac{d}{d y} \rho_{i}(q, y)\right) C^{\prime}(y) .
$$

So whenever $\frac{d}{d y} \rho_{i}(q, y) \neq 1$ it holds $\left(C_{(\xi, \gamma),-i}\right)^{\prime}\left(y-\rho_{i}(q, y)\right)=C^{\prime}(y)$. This is exactly what we need since now

$$
\begin{aligned}
\mu_{N \backslash i}^{\rho}(q, C) & =\int_{0}^{q(N)} C^{\prime}(t) \mathrm{d} \rho_{N \backslash i}(q, t)= \\
& =\int_{0}^{q(N)} C^{\prime}(t) \mathrm{d} \rho\left(q_{N \backslash i}, t-\rho_{i}(q, t)\right)= \\
& =\int_{0}^{q(N)}\left(C_{(\xi, \gamma),-i}\right)^{\prime}\left(t-\rho_{i}(q, t)\right) \mathrm{d} \rho\left(q_{N \backslash i}, t-\rho_{i}(q, t)\right)= \\
& =\int_{0}^{q(N \backslash i)}\left(C_{(\xi, \gamma),-i}\right)^{\prime}(t) \mathrm{d} \rho\left(q_{N \backslash i}, t\right)=\mu^{\rho}\left(q_{N \backslash i}, C_{(\xi, \gamma),-i}\right) .
\end{aligned}
$$

Weak consistency as a property for mechanism $\mu$ is as appealing as the contract supporting it. Recall that there are no severe built-in requirements on contracts, so that we may find mechanisms weakly consistent although there is in practice no good reason to assume that agents will agree to the underlying contract. So weak consistency as a relaxation of consistency takes too extreme a position. Now we will look for a more restrictive notion than weak consistency by demanding the existence of special types of contracts under which solutions to the induced reductions are stable.

Denote for $C \in \mathcal{C}$ the right derivative at $t \in \mathbb{R}_{+}$by $D^{+} C(t)$.

\section{Upperbound}

Contract $(\xi, \gamma)$ for $\mu$ satisfies upperbounds if for all $(q, C) \in \mathcal{P}^{N}, y \in[0, q(N)], i \in N$

$$
\gamma_{i}(y, q, C) \leq \xi_{i}(y, q, C) \cdot \sup _{t \in[0, q(N)]} D^{+} C(t) .8
$$

The idea is that a contract conflicting with upperbounds will make individual agents want to revoke it. Call contract $(\xi, \gamma)$ for $\mu$ regular if it satisfies upperbounds. Note that $\mu$ satisfies

\footnotetext{
${ }^{7}$ Here I use the fact that a monotonic rationing method $\rho$ is Lipschitz-continuous and - in particular absolutely continuous in the resource component $t$.

${ }^{8}$ Here other ideas come to mind. Also that $\gamma(y, q, C)$ be contained in the pessimistic imputation set $I^{*}(\xi(y, q, C))$ for all $(q, C) \in \mathcal{P}^{N}, y \in[0, q(N)]$. I will use the property only for the case $C=B_{x}$, and here both types of upperbound lead to the same conclusions.
} 
upperbounds as well, by subsitution of $y=q(N)$ in (16). Clearly any such $\mu$ satisfies constant returns.

\section{Regular consistency}

There is at least one regular contract $\varphi$ for $\mu$ such that $\mu_{N \backslash i}(q, C)=\mu\left(q_{N \backslash i}, C_{\varphi,-i}\right)$ for all $(q, C) \in \mathcal{P}^{N}, i \in N$.

Lemma 5.3 Consider a regular contract $(\xi, \gamma)$ for mechanism $\mu$. Then for all $x \in \mathbb{R}_{+}, i \in N$

$$
\left(B_{x}\right)_{(\xi, \gamma),-i}=B_{x-\gamma_{i}\left(q(N), q, B_{x}\right)} .
$$

Proof. Take $x \in[0, q(N)]$. We have by definition of the reduced cost function

$$
\left(B_{x}\right)_{(\xi, \gamma),-i}\left(k-\xi\left(k, q, B_{x}\right)\right)= \begin{cases}k-\gamma_{i}\left(k, q, B_{x}\right) & \text { if } k \leq x, \\ x-\gamma_{i}\left(k, q, B_{x}\right) & \text { if } k \in(x, q(N)] .\end{cases}
$$

Firstly, in order to qualify as a cost function we must have that on $(x, q(N)]$ the mapping $k \mapsto x-\gamma_{i}\left(k, q, B_{x}\right)$ is non-decreasing. But at the same time $k \mapsto \gamma\left(k, q, B_{x}\right)$ is non-decreasing. Then $k \mapsto x-\gamma_{i}\left(k, q, B_{x}\right)$ must be constant on $(x, q(N)]$, and in particular $x-\gamma_{i}\left(k, q, B_{x}\right)=$ $x-\gamma_{i}\left(q(N), q, B_{x}\right)$ for all $k \in(x, q(N)]$.

Secondly, regularity implies for $y \leq x, \gamma\left(y, q, B_{x}\right) \leq \xi\left(y, q, B_{x}\right)$. Also by definition of $\gamma$ and $\xi$ we have $\sum_{j \in N} \gamma_{j}\left(y, q, B_{x}\right)=B_{x}(y)=y=\sum_{j \in N} \xi_{j}\left(y, q, B_{x}\right)$, hence $\gamma\left(y, q, B_{x}\right)=\xi\left(y, q, B_{x}\right)$.

Then (17) turns into

$$
\left(B_{x}\right)_{(\xi, \gamma),-i}(y)= \begin{cases}y & \text { for } y \in\left[0, x-\gamma_{i}\left(q(N), q, B_{x}\right),\right. \\ x-\gamma_{i}\left(q(N), q, B_{x}\right) & \text { if } y>x-\gamma_{i}\left(q(N), q, B_{x}\right) .\end{cases}
$$

Within the context of Theorem 4.1, regular consistency smoothly transfers from the cost sharing context to the rationing model and back. The mappings $r \mapsto \mu^{r}$ and $\mu \mapsto r^{\mu}$ with $r^{\mu}(q, t)=\mu\left(q, B_{t}\right)$ define an isomorphism between the set of consistent rationing methods and $M(\mathrm{ADD}, \mathrm{RCONS})$.

Theorem $5.4 \mu \in M(\mathrm{ADD}, \mathrm{RCONS})$ if and only if $\mu=\mu^{r}$ and $r$ is a consistent monotonic rationing method.

Proof. Take $\left(q, B_{x}\right) \in \mathcal{P}^{N}, i \in N$. Since $\mu=\mu^{r}$ is consistent there is a regular contract $(\xi, \gamma)$ such that $\mu_{N \backslash i}\left(q, B_{x}\right)=\mu\left(q_{N \backslash i},\left(B_{x}\right)_{(\xi, \gamma),-i}\right)$. In particular $\gamma_{i}\left(q(N), q, B_{x}\right)=\mu_{i}\left(q, B_{x}\right)=r_{i}(q, x)$. By application of Lemma $5.3\left(B_{x}\right)_{(\xi, \gamma),-i}=B_{x-\gamma_{i}\left(q(N), q, B_{x}\right)}=B_{x-r_{i}(q, x)}$. So

$$
\mu\left(q_{N \backslash i},\left(B_{x}\right)_{(\xi, \gamma),-i}\right)=\mu\left(q_{N \backslash i}, B_{x-r_{i}(q, x)}\right)=r\left(q_{N \backslash i}, x-r_{i}(q, x)\right) .
$$


Then it holds $r_{N \backslash i}(q, x)=\mu_{N \backslash i}\left(q, B_{x}\right)=\mu\left(q_{N \backslash i},\left(B_{x}\right)_{(\xi, \gamma),-i}\right)=r\left(q_{N \backslash i}, x-r_{i}(q, x)\right)$. By variation over all rationing problems $(q, x)$ we conclude that $r$ is consistent.

Now the other way, assume $r$ is a consistent and monotonic rationing method and let $\mu=\mu^{r}$. Define a regular contract $(\xi, \gamma)$ for $\mu$ by $\xi(y, q, C)=r(q, y)$ and $\gamma(y, q, C)=\int_{0}^{y} C^{\prime}(t) \mathrm{d} r(q, t)$ and continue the proof as in Theorem 5.2.

The result shows in particular that under additivity, RCONS is the same as the consistency notion discussed in Koster (2007).

Interestingly, $\mu^{\mathrm{SR}}$ may be not consistent, but it is regularly consistent as it relates to a consistent rationing method $r^{\mathrm{UG}}$. Also the priority rationing methods $r^{\sigma}$ are consistent and so $\mu^{\sigma}$ is regularly consistent. Compare this with $\mu^{\mathrm{ss}}$, which is not regularly consistent, since its natural counterpart in rationing, $r^{\mathrm{RP}}$, is not.

Conjecture 5.5 $M(\mathrm{ADD}, \mathrm{RCONS})=M(\mathrm{ADD}, \mathrm{CR}, \mathrm{WCONS})$

Example 5.6 The properties RCONS and ADD are logically independent. Tijs and Koster (1998) relate to each $P=(q, C) \in \mathcal{P}^{N}$ a pessimistic cost sharing problem $\left(q, C_{P}^{*}\right)$, where the pessimistic cost function $C_{P}^{*}$ is defined as the concave rearrangement of $C$ on $[0, q(N)]$,

$$
C_{P}^{*}(y)= \begin{cases}\sup \left\{\int_{T} C^{\prime}(t) \mathrm{d} t \mid T \subseteq[0, q(N)], \lambda(T)=y\right\} & \text { if } y \in[0, q(N)], \\ C(y) & \text { if } y>q(N),\end{cases}
$$

Tijs and Koster (1998) shows that for any $P=(q, C) \in \mathcal{P}^{N}$ there is a family $\left\{T_{P}(x)\right\}_{x \in[0, q(N)]}$ of increasing and $\lambda$-measurable subsets such that $\lambda(T(x))=x$ and $\int_{T(x)} C^{\prime}(t) \mathrm{d} t=C_{P}^{*}(x)$ for all $x \in[0, q(N)]$. Now consider the pessimistic marginal mechanism $\mu^{\sigma, *}$, defined for $P=(q, C) \in$ $\mathcal{P}^{N}$ by $\mu^{\sigma, *}(q, C):=\mu^{\sigma}\left(q, C_{P}^{*}\right)$. Define $U_{i}=T\left(q_{i}+\sum_{j: \sigma(j)<\sigma(i)} q_{j}\right) \backslash T\left(\sum_{j: \sigma(j)<\sigma(i)} q_{j}\right)$ and a contract $(\xi, \gamma)$ for $\mu^{\sigma, *}$ by $\xi_{i}(y, q, C)=\int_{0}^{y} \mathbb{I}_{U_{i}}(t) \mathrm{d} t, \gamma_{i}(y, q, C)=\int_{0}^{y} C^{\prime}(t) \mathbb{I}_{U_{i}}(t) \mathrm{d} t$. Then this contract may be used to show that $\mu^{\sigma, *}$ is regularly consistent. Clearly $\mu^{\sigma, *}$ is not additive. $\triangleleft$

Having established the tight relationship between consistent rationing and regularly consistent cost sharing, we may find other characterizations of cost sharing mechanisms by known characterizations of corresponding rationing methods. I will give one such an example, based on the characterization of parametric rationing by Young (1987).

A rationing method $r$ is called continuous if it is jointly continuous in both arguments, i.e., $(q, t) \mapsto r(q, t)$ is continuous for all rationing problems $(q, t)$. Such $r$ is then robust against small changes in the parameters defining the rationing problem. For cost sharing mechanisms 
the approach is similar; a mechanism will be called continuous if small changes in demands and $B_{t}$ cause only small changes in cost shares. More specifically,

Continuity (CONT) The mapping $(q, t) \mapsto \mu\left(q, B_{t}\right)$ is continuous on $\mathbb{R}_{+}^{S} \times \mathbb{R}_{+}$, for all $S \subseteq N .^{9}$

Theorem 5.7 $\mu \in M(\mathrm{ADD}, \mathrm{RCONS}, \mathrm{ET}, \mathrm{CONT})$ if and only if $\mu=\mu^{r}$ for some continuous parametric $r$.

Proof. Take $\mu$ with the listed properties. Then the rationing method $r$ defined by $r(q, t):=$ $\mu\left(q, B_{t}\right)$ satisfies continuity (by definition), consistency, and equal treatment. Since we have an infinite pool $\mathcal{N}$ of agents we can apply Theorem 1 in Young (1987), which implies that $r$ is continuous and parametric. Moulin and Shenker (1994) show that $\mu=\mu^{r}$. Now take a continuous parametric $r$. By definition $\mu^{r}$ is continuous. Moreover, since $r$ satisfies equal treatment, so does $\mu^{r}$ by Theorem 4.3. Finally, $r$ is consistent by Theorem 1 in Young (1987), so we are done by referring to Theorem 5.4.

5.1. Consistent extensions. Given a cost sharing mechanism defined for only 2-agent problems, it natural to ask whether it is possible to extend it in a consistent way to a mechanism for arbitrarily finite sets of agents. The related problem for rationing methods has been partially solved. Dagan and Volij (1997) show that any rationing method for 2-agent problems allows for a unique average consistent method. And, moreover, whenever a consistent extension exists, it must coincide with the average consistent method. In particular this shows that there is at most one possibility to extend a rationing method for 2-agent problems in a consistent way. An example of a rationing method for 2-agent problems which cannot be extended consistently is the claims-truncated proportional rule (see Thomson (2008)). Then Theorem 5.4 implies:

Theorem 5.8 Let $\bar{\mu}$ be an additive mechanism with the property $\mathrm{CR}$ defined for 2-agent cost sharing problems only. Then there is at most one way to extend $\bar{\mu}$ to $\mu \in M(\mathrm{ADD}, \mathrm{RCONS})$.

For instance, this shows that there is at most one mechanism $\mu \in M(\mathrm{ADD}, \mathrm{RCONS})$ extending serial- or average cost sharing for 2-agent problems, whereas there is no consistent extension of the mechanism defined through the truncated claims proportional method.

In principle, consistent cost sharing mechanisms can be constructed using the techniques of Dagan and Volij (1997). Once the rationing method underlying the mechanism for 2-agent problems is known, calculate the corresponding average consistent extension and - if at all the consistent mechanism results from Theorem 5.4.

\footnotetext{
${ }^{9}$ In order to avoid the hybrid character of CONT one may consider the replacement by two requirements, continuity of the mappings $t \mapsto \mu\left(q, B_{t}\right)$ and $q \mapsto \mu\left(q, B_{t}\right)$.
} 


\section{Discussion AND OPEN PROBLEMS}

Instead of taking cost sharing mechanisms as the basic tool to share costs, I could have started with contracts as the primitive notion of solution; all could have been written in terms of constracts, since any contract defines a cost sharing mechanism in an unambiguous way. I have chosen not to do so in order to stick to study the notion of consistency in the standard framework.

I reflected on the fact that consistency may be valued contingent on the quality of the underlying contracts. I started of with the two diametrically opposed notions of consistency, and discussed regular consistency as a reasonable intervening notion. The idea is that mechanisms with many regular contracts are desirable, and that for the same reason it may be easier for these to comply with regular consistency. However, another way to proceed is to demand for consistency with respect to all regular contracts. Notice the tension between the pluriformity of regular contracts and the corresponding notion of consistency: on the one hand it will be easier to meet existence of regular contracts, but on the other hand, the more regular contracts exist, the more demanding consistency gets. The situation becomes even more complex if revision of the idea of a regular contract is considered; the definition is rather ad-hoc now. Further study on these refinements is needed to shed more light on the structure of the space of solutions meeting these new standards.

Also this may lead to a better understanding so that a couple of open problems arising in this paper can be answered. First there is Conjecture 5.5, which states that under additive cost sharing and constant returns weak consistency is equivalent to regular consistency. Another open problem is whether a nonadditive and consistent mechanism $\mu$ can be found which meets independence from irrelevant cost, i.e., $\mu\left(q, C_{1}\right)=\mu\left(q, C_{2}\right)$ if only $C_{1}(y)=C_{2}(y)$ for all $y \geq q(N)$. If not, then this would lead to the conclusion that consistency and independence form irrelevant cost together imply additivity, see, e.g., example 4.10.

\section{REFerences}

1. M. J. Albizuri and J.M Zarzuelo, The dual serial cost-sharing rule, Mathematical Social Sciences 53 (2007), 150-163.

2. R. J. Aumann and M. Maschler, Game theoretic analysis of a bankruptcy problem from the Talmud, Journal of Economic Theory 36 (1985), 195-213.

3. N. Dagan and O. Volij, Bilateral comparisons and consistent fair division rules in the context of bankruptcy problems, International Journal of Game Theory 26 (1997), 11-25.

4. M. Davis and M. Maschler, The kernel of a cooperative game, Naval Research Logistic Quarterly 12 (1965), 223-259.

5. S. Hart and A. Mas-Colell, Potential, value and consistency, Econometrica 57 (1989), 589-614. 
6. J.L. Hougaard and L. Thorlund-Petersen, Mixed serial cost sharing, Mathematical Social Sciences 41 (2001), $51-68$.

7. M. Kaminski, 'Hydrolic' rationing, Mathematical Social Sciences 40 (2000), 131-155.

8. M. Koster, Concave and convex serial cost sharing, Chapters in Game Theory (Dordrecht, The Netherlands) (P. Borm and H. Peters, eds.), Kluwer Academic Press, 2002.

9. S Sharing variable returns of cooperation, CeNDEF Working Paper 05-06, University of Amsterdam, The Netherlands, 2005.

10. _ Consistent cost sharing, mimeo, University of Amsterdam, The Netherlands, 2007.

11. M. Maschler, Consistency, Game Theory and Applications (New York) (T. Ichiishi, A. Neyman, and Y. Tauman, eds.), Academic Press, 1990, pp. 183-186.

12. L. Massoulié and J.W. Roberts, Bandwith sharing: Objectives and algorithms, in Proceedings of IEEE INFOCOM Conference (1999), 1395-1403. IEEE/ACM Transactions on Volume 10, Issue 3, Jun 2002 Page(s):320 - 328

13. H. Moulin, Equal or proportional division of a surplus, and other methods, International Journal of Game Theory 16 (1987), 161-186.

14. _ Priority rules and other asymmetric rationing methods, Econometrica 68 (2000).

15. __ Axiomatic cost and surplus-sharing, Handbook of Social Choice and Welfare (Amsterdam) (K. J. Arrow, A. K. Sen, and K. Suzumura, eds.), Handbooks in Economics 19, North-Holland Elsevier, 2002, pp. 289-357.

16. H. Moulin and S. Shenker, Serial cost sharing, Econometrica 60 (1992), 1009-1037.

17. H. Moulin and S. Shenker, Average cost pricing versus serial cost sharing; an axiomatic comparison, Journal of Economic Theory 64 (1994), 178-201.

18. B. O'Neill, A problem of rights arbitration from the Talmud, Mathematical Social Sciences 2 (1982), 345371.

19. W. Sharkey, The Theory of Natural Monopoly, Cambridge University Press, Cambridge, U.K., 1985.

20. M. Shubik, Incentives, decentralized control, the assignment of joint cost, and internal pricing, Management Science 8 (1962), 325-343.

21. A. I. Sobolev, The functional equations that give the payoffs of the players in an n-person game, Advances in Game Theory (E. Vilkas, ed.), Izdat. "Mintis," Vilnius, 1973, pp. 151-153.

22. Y. Sprumont, Ordinal cost sharing, Journal of Economic Theory 81 (1998), 126-162.

23. P. Sudhölter, Axiomatizations of game theoretical solutions for one-output cost sharing problems, Games and Economic Behavior 24 (1998), 42-71.

24. W. Thomson, On the axiomatic method and its recent applications to game theory and resource allocation, Social Choice and Welfare 18 (2001), 327-386.

25. __ Axiomatic and game-theoretic analysis of bankruptcy and taxation problems: a survey, Mathematical Social Sciences 45 (2003), 249-297.

26. Consistent allocation rules, Mimeo, Economics Department, University of Rochester, Rochester, U.S.A., 2006.

27. _ The two-agent claims-truncated proportional rule has no consistent extension: a constructive proof, Economics Letters 98 (2008), 59-65.

28. S. H. Tijs and M. Koster, General aggregation of demand and cost sharing methods, Annals of Operations Research 84 (1998), 137-164.

29. C. Trudeau, Cost sharing with multiple technologies (2009), mimeo, Université de Montréal, Montréal.

30. R. J. Weber, Probabilistic values for games, The Shapley Value (A. E. Roth, ed.), Cambridge University Press, Cambridge, 1988. 
31. H. P. Young, Cost allocation: Methods, Principles, Applications, North-Holland, Amsterdam, The Netherlands, 1985 .

32. __ On dividing an amount according to individual claims or liabilities, Mathematics of Operations Research 12 (1987), 398-414.

33. _ Distributive justice in taxation, Journal of Economic Theory 44 (1988), 321-335.

34. _ Cost allocation, Handbook of Game Theory II (R.J. Aumann and S. Hart, eds.), Elsevier, Amsterdam, The Netherlands, 1994, pp. 1193-1235.

(M. Koster) CendeF, Department of Quantitative Economics, University of Amsterdam Roetersstraat 11, 1018WB Amsterdam, The Netherlands

E-mail address, Maurice Koster: mkoster@uva.nl 